\title{
A Formal Approach to Distance-Bounding RFID Protocols
}

\author{
Ulrich Dürholz ${ }^{2} \quad$ Marc Fischlin ${ }^{1} \quad$ Michael Kasper $^{2} \quad$ Cristina Onete $^{1}$ \\ ${ }^{1}$ Darmstadt University of Technology \& CASED, Germany \\ www.minicrypt.de \\ ${ }^{2}$ Fraunhofer Institute for Secure Information Technology (SIT) and CASED, Germany
}

\begin{abstract}
Distance-Bounding identification protocols aim at impeding man-in-themiddle attacks by measuring response times. There are three kinds of attacks such protocols could address: (1) Mafia attacks where the adversary relays communication between honest prover and honest verifier in different sessions; (2) Terrorist attacks where the adversary gets limited active support from the prover to impersonate. (3) Distance attacks where a malicious prover claims to be closer to the verifier than it actually is. Many protocols in the literature address one or two such threats, but no rigorous cryptographic security models - nor clean security proofs - exist so far. For resource-constrained RFID tags, distance-bounding is more difficult to achieve. Our contribution here is to formally define security against the above-mentioned attacks and to relate the properties. We thus refute previous beliefs about relations between the notions, showing instead that they are independent. Finally we use our new framework to assess the security of the RFID distance-bounding scheme due to Kim and Avoine, and enhance it to include impersonation security and allow for errors due to noisy channel transmissions.
\end{abstract}

Keywords. RFID distance-bounding protocols, formal models, provable security

\section{Introduction}

Man-in-the-middle attacks are a powerful strategy for an adversary to fool identification schemes: by relaying communication between provers and verifiers, the adversary makes verifiers accept. Following [16] quintessential relaying is called Mafia fraud. Environments with no central authority and certificates, like RFID identification, are particularly subject to such attacks. Practical set-ups [23, 15, 17, 22, 19] indicate their feasibility, and several works investigate attacks on the HB protocol $[27,20,18,9,32,30]$, which is designed for low-power devices e.g. RFIDs. For a more general overview of RFID security issues see [28]. 


\subsection{Distance-Bounding Protocols}

Distance-bounding protocols, proposed initially by Brands and Chaum [8], suggest a countermeasure against man-in-the-middle attacks. The basic idea is that relaying communication takes longer than genuine reponses. Thus, if verifiers measure the time elapsed between sending a value and receiving the reply, man-in-the-middle attacks should become infeasible. In practice, verifiers check round-times for many so-called fast or time-critical communication phases, (as opposed to slow or lazy phases, where round times do not matter).

We mainly address RFID authentication, but our new framework applies to general distance-bounding protocols where provers and verifiers may interact and at the end the verifier outputs a bit indicating whether the prover has been authenticated or not. For RFID authentication, the verifiers are readers and the provers are RFID tags; we use these terms interchangeably for provers and verifiers. RFID distance-bounding has been investigated quite extensively $[1,2,4,8,10,12,13,15,16,17,22,23,26,29,34,36,3,37]$. See also [24] for a comprehensive overview. The three main threats that need to be avoided are: (1) Mafia fraud, where the adversary tries to impersonate to the reader while communicating with the genuine tag (the timing prevents it from using pure relay though); (2) Terrorist fraud where tags may leak useful information to the adversary in offline phases to help it authenticate (the restriction being that tags should not reveal trivial information like the secret key); (3) Distance Fraud, where the tag claims to be closer than it actually is. We also consider the basic (often neglected) requirement for identification, i.e. slow-round impersonation resistance, independent of the limited number of fast phases.

We exemplify the three attacks as follows: consider a gym locker with an inbuilt RFID reader, for which Alice holds the unique pass key (an RFID tag). One evening, Alice is not at the gym, but at a party. In the Mafia fraud scenario, Bob is at the gym; his accomplice, Bobette, is at the party with Alice. Bob wants to open the locker (without Alice's consent for Mafia fraud). In this attack, Bob and Bobette relay messages between the locker and Alice's tag. If, on the contrary, Alice wants Bob to use her locker (for this night only) we have Terrorist fraud. Alice may now give Bob information to help him use her locker, but she doesn't want Bob to abuse her kindness and open the locker on his own, this or any other time. For Terrorist attacks thus, Alice helps Bob herself: Bobette is not needed. Finally, if Alice parked her car in a bad spot, she might want to "prove" that she was at the gym instead (this is distance fraud) by opening the locker, which can be opened only if the unique key is in direct proximity.

Several existing protocols implement resistance against one (or more) of the above threats. A selection of such protocols is compared in Figure 1. The values mentioned for $[8,26,4,34,29]$ are those claimed by the respective papers (despite a lack of formal approaches). We note that public-key constructions, as opposed to private-key ones, are unsuitable for low-power devices like RFID. Also, most existing work permits adversaries to impersonate the reader to the tag, thus leaking information about fast-phase response times. If only bits are transmitted in fast phases, the ideal impersonation bound would be 
$2^{N_{c}}$ for $N_{c}$ critical rounds; however, most protocols allow impersonation and thus reach a lower than ideal bound. To account for this Mafia fraud attack, under "Rounds", we give the number $N_{c}$ of time-critical rounds required for a Mafia resistance of about $2^{-k}$. We round down the number of rounds in $[26,34]$ to $2 k$. Note that [4] shows a construction with reduced complexity, at the expense of security.

\begin{tabular}{|c|c|c|c|c|c|}
\hline & {$[\mathbf{8}]$} & {$[\mathbf{2 6}]$} & {$[\mathbf{4}]$} & {$[\mathbf{3 4}]$} & {$[\mathbf{2 9}]$} \\
\hline Mafia & $\checkmark$ & $\checkmark$ & $\checkmark$ & $\checkmark$ & $\checkmark$ \\
Terror & $\times$ & $\times$ & $\times$ & $(\checkmark)^{1}$ & $\times$ \\
Distance & $\checkmark$ & $\checkmark$ & $\checkmark$ & $\checkmark$ & $\checkmark$ \\
Impersonation & $\times$ & $\times$ & $\checkmark$ & $\times$ & $\times$ \\
Rounds $N_{c}$ & $k$ & $>2 k$ & $k$ & $>2 k$ & $k$ \\
Storage & $N_{c}$ & $2 N_{c}$ & $O\left(2^{N_{c}}\right)$ & $2 N_{c}$ & $4 N_{c}$ \\
Private-key & $\times$ & $\checkmark$ & $\checkmark$ & $\checkmark$ & $\checkmark$ \\
\hline
\end{tabular}

Figure 1: Claimed Security and Actual Efficiency of Distance Bounding Protocols at a glance ( ${ }^{1}$ only special terrorists, no formal proof.)

We lastly outline some related cryptographic concepts from the literature. Most prominently, the recent position-based cryptography work [14] aims to determine if a prover is (exactly) at a claimed position — but in a single protocol run, with many verifiers. This is clearly different from Mafia fraud or terrorist fraud attacks. As adversaries in [14] must all have the same knowledge as the prover (also knowledge of the private key), this model is closest to our distance fraud model, where tags must prove they are closer to the reader than they really are. However, exact positioning is impossible in practice for RFID, requiring too many readers to deal with the high variance in response time. ${ }^{1}$

By contrast, self-delegation as in [21] and [11] resembles terrorist fraud. In [21], secondary, self-delegated keys are used to authenticate; however losing many of them compromises the long-term key, as in our idea of terrorist fraud where the malicious tag reveals part of the key by helping the man-in-the-middle. The main differences in the model are: that [21] consider the public-key setting only (with server certification of secondary keys), that they investigate signature-leakage only, and that no online help (with restriction due to the distance) is available. Also, [21] relies on public-key cryptography and non-interactive zero-knowledge proofs, primitives that are unsuitable for RFID.

Finally [11] model transferability of anonymous credentials. This "all-or-nothing" approach associates sharing secret information (pseudonyms or credentials only) to recovery of users' full secret. This is again similar to terrorist resistance but [11] do not formally model attacks and security. The use of public-key infrastructures here also makes the idea inapplicable to RFID.

\footnotetext{
${ }^{1}$ Recent work due to Hancke [25] in fact suggests designing a distance bounding channel limiting channel-specific variations of response times.
} 


\subsection{Our Contributions}

Our contributions are threefold: (1) We give rigorous cryptographic security models for Mafia, Terrorist, and Distance fraud, thus (2) relating the security properties formally. We also refute the claim in [34] that terrorist attacks resistance implies distance-fraud resistance. Finally, we (3) use our framework to formally assess the security of the prominent scheme in [29], and enhance it to allow for noisy channels and implement impersonation resistance.

The Practice behind the Theory. Practical investigations [13, 15, 34, 26, 33, 25] indicate some design issues for RFID distance-bounding protocols. As such considerations apply for all low-power devices, we provide for them in our framework. Firstly, measuring round-trip time to send multiple bits is dangerous $[15,25,34]$ as transmissions become more unreliable and have fresh noise. In practice thus, readers and tags must exchange only bits in time-critical phases. Also, time-critical computations must be simple and should take consistent time, so as not to strongly bias round-trip time and threshold errors. Distance-bounding protocols for low-power devices like RFID should use little storage and provide for noise both in transmissions and in time measurement $[15,25,34]$. Our model introduces thresholds for failures during timed steps, adding depth to the framework and allowing the adversary to relay communication for some phases.

Lastly, implementations may allow adversaries to predict a bit "halfway into the signal" [17]. Also, computation complexity may vary with received input, and adversaries can get information from the reader or tag faster than expected. Our model allows the adversary to relay data as long as it is not purely duplicated. Also, note that often authentication in distance bounding is restricted only to the few fast communication rounds supported by the tag [4]. We suggest using offline authentication, preferable preceding fast phases - as suggested in [4]. Some protocols do not have this property $[8,26]$, whereas we give a strong definition of it and suggest it as an enhancement of protocols like [29].

The Models. A sound modeling of the above attacks is crucial to assessing protocol security. Confusions appear especially with attack modes and successful man-in-themiddle attacks, e.g. for the HB protocol [27, 20, 18, 9, 32, 30]. As another example, the allegedly secure Hitomi and NUS protocols were recently proved insecure [1]. We formalize game-based models while also considering practical conditions. This enables us to formally prove that, contrary to the remark of [34], terrorist fraud resistance does not imply distance fraud resistance. In fact, we show that Mafia resistance, terrorist resistance, and distance-fraud resistance are all independent. More precisely, we present protocols that are vulnerable to one attack, but resistant to all others (including the basic authentication-protocol requirement of impersonation resistance). In particular, terrorist fraud resistance also does not imply Mafia fraud resistance, nor vice versa.

Avoine et al. [2] already laid a concurrent groundwork; our approach here is more formal and rigorous, based on the common game-based notion in cryptography. Due to 
our formalization, we prove (contrary to the statements of [2]) that the three types of fraud are independent. Also, [31] shows a formal approach, but against honest provers only and without specifying security goals. Finally, the formal methods approach in [35] thoroughly models distance bounding with formal methods, but treats wireless networks in general, assuming that provers and verifiers have equal capacities (unlike RFID systems, where tags are computationally weaker). Additionally, some physical properties of RF communication, such as the unreliability of tags' backscattering and colliding signals, are unaccounted for. From a cryptographic point of view they do not provide reliable definitions for the different kind of attacks discussed above.

Using our Framework. We use our framework to assess the security of the protocol due to Kim and Avoine [29], which relies on mutual tag-to-reader and reader-to-tag fastphase authentication to achieve good Mafia and Distance Fraud (but not impersonation-) resistance. If reader authentication fails, the tag generates random responses every round. We first make the construction in [29] impersonation resistant, then formally assess its security in our framework. We also prove that it is not terrorist-fraud resistant in our model.

\section{Preliminaries}

We consider a single reader $\mathcal{R}$ and a single tag $\mathcal{T}$, sharing a secret key generated through $\mathrm{Kg}$. To the reader we associate a clock and a database entry storing the tag's secret key. We assume that the identification scheme $\mathcal{I D}=(\mathrm{Kg}, \mathcal{T}, \mathcal{R})$ marks (consecutive) steps of the identification protocol as lazy or time-critical: in time-critical steps, one party - usually the reader - measures the round-time $\Delta t$ and compares it to a predetermined threshold $t_{\mathrm{max}}$; else the phase is called lazy. A protocol run can consist of arbitrary nonoverlapping sequences of lazy and time-critical phases, with time-critical phases possibly following one another. Denote by $N_{c}$ the number of time-critical phases. Errors due to time-measurement noise are modelled by allowing $T_{\max }$-many round-times to exceed $t_{\max }$. Similarly, $E_{\max }$ is the maximum number of time-critical phases with erroneous transmissions.

Definition 2.1 An identification scheme for timing parameters $\left(t_{\max }, T_{\max }, E_{\max }\right.$, $\left.N_{c}\right)$ is a triplet of efficient algorithms $\mathcal{I D}=(\mathrm{Kg}, \mathcal{T}, \mathcal{R})$ with:

Key Generation. For parameter $n \in \mathbb{N}, \mathrm{Kg}$ generates a secret key sk.

IDENTIFICATION. The joint execution of algorithms $\mathcal{T}(s k)$ and $\mathcal{R}(s k)$ generates, depending on $t_{\max }, T_{\max }, E_{\max }, N_{c}$, a verifier output $b \in\{0,1\}$.

We assume that the scheme is complete: for any $n \in \mathbb{N}$ and any key sk $\leftarrow \operatorname{Kg}\left(1^{n}\right)$, the decision bit b produced by honest party $\mathcal{R}(s k)$ interacting with honest party $\mathcal{T}($ sk) under the requirements following from the timing parameters, is 1 with probability (negligibly close to) 1. 
Note that although most definitions of distance-bounding protocols omit $t_{\max }$, this parameter is a crucial difference between distance-bounding and authentication protocols, where $t_{\max }$ is by default infinitely large. The parameters $E_{\max }$ and $T_{\max }$ are intrinsic to communication over noisy channels (e.g. RF channels between readers and passive and semi-passive RFID tags ${ }^{2}$ ). In distance bounding, it is unreasonable to separate the reliability of the communication from its security; these properties are connected by the importance of round-time measurements towards acceptance or rejection. Bit errors are unavoidable in RF communication, as stated in point 4 of Clulow et al.'s principles for secure time-of-flight distance-bounding [15]. As described in section 1, RF communication noise implies that transmissions between readers and tags are not always reliable, possibly reaching the reader outside the time bound. We can, however, set $T_{\max }=E_{\max }=0$ for extremely reliable scenarios.

\section{Security Model}

\subsection{Communication Model}

The adversary can access: a reader instance to which it impersonates the tag (a readeradversary session), a tag instance to which it impersonates the reader (adversary-tag session), and an interface observing a genuine reader-tag protocol for which the adversary cannot change transmissions (reader-tag session). The adversary can access all interfaces concurrently and in many sessions (sessions share a secret key, but have different random tapes). Each session has an identifier sid (given to the adversary, but not to protocol participants). We assume that the adversary knows if an authentication attempt succeeded or not. ${ }^{3}$

In our concurrent single-reader-single-tag scenario (as opposed to a single reader and multiple tags), many instances of the single tag may exist in parallel, sharing the secret key, but not the random tape. The key is static, i.e., not updated after executions. For many independent keys (multiple tags), adversaries can always pick a tag to attack in our model. Three factors are crucial to multiple-tag scenarios: the interdependency of the keys; the noise in the communication due to tag-to-reader collisions (a factor modeled by $\left.E_{\max }\right)$; and key management. A formal approach for key update is, however, beyond the scope of this paper.

We assume message-driven attacks, i.e., honest parties reply as soon as they receive a (protocol) message. The adversary schedules message delivery to honest parties. We assume a global clock, assigning an integer clock(sid, $k$ ) to the $k$-th protocol message, delivered in session sid to an honest party. The honest party's reply is assigned $\operatorname{clock}(\operatorname{sid}, k+1)=\operatorname{clock}(\operatorname{sid}, k)+1 .^{4}$ Furthermore, $\operatorname{clock}(\operatorname{sid}, k)<\operatorname{clock}\left(\operatorname{sid}^{*}, k\right)$ if the

\footnotetext{
${ }^{2}$ Passive RFID tags have no power source of their own and are very sensitive to their environment, in particular metals and liquids. Semi-passive tags use their own power source for computation, but rely on readers for communication, and are also vulnerable to interference by metals and liquids.

${ }^{3}$ This is not a strong requirement. In practice the success of an authentication attempt is marked by a physical event: a beep, the opening of a door, a green light etc.

${ }^{4}$ We could also allow adversaries to delay message delivery from honest parties. Our model and results
} 
adversary delivers the $k$-th message in session sid* after the $k$-th message in session sid. Denote by $\Pi_{\text {sid }}[i \ldots j]$ messages $i$ to $j$ exchanged in session sid and by $\Pi_{\text {sid }}[1 \ldots]$ all messages exchanged in sid. Let view $\mathcal{A}_{\mathcal{A}}$ denote the adversary's view in an attack, containing its internal randomness and all the transcripts (of communication with and among other parties).

Let $t$ denote the adversary's running time, including steps of honest parties. Denote by $q_{\mathcal{R}}$ (resp. $q_{\mathcal{T}}$ and $q_{\mathrm{OBS}}$ ) the maximal number of reader-adversary (resp. adversary-tag and reader-tag) sessions. Below we refine the attacks and define winning conditions for the adversary (who must non-trivially impersonate the tag in a reader-adversary session). For an attack att we write $\mathbf{A d v}_{\mathcal{I D}}^{\text {att }}(\mathcal{A})$ for the probability that the $\left(t, q_{\mathcal{R}}, q_{\mathcal{T}}, q_{\mathrm{OBS}}\right)$-adversary $\mathcal{A}$ wins.

\subsection{Mafia Fraud Detection Model}

Mafia fraud adversaries can communicate arbitrarily with tag and reader, except for purely relaying time-critical transmissions. We exclude only attacks where the adversary relays exact transmissions, calling such time-critical phases tainted:

Definition 3.1 (Tainted Time-Critical Phase (Mafia)) A time-critical phase $\Pi_{\text {sid }}[k \ldots k+$ $2 \ell-1]=\left(m_{k}, \ldots, m_{k+2 \ell-1}\right)$ for $k, \ell \geq 1$ of a reader-adversary session sid, with the $k$-th message being received by the adversary, is tainted by the phase $\Pi_{\text {sid }^{*}}[k \ldots k+2 \ell-1]=$ $\left(m_{k}^{*}, \ldots, m_{k+2 \ell-1}^{*}\right)$ of an adversary-tag session sid* if for all $i=0,1, \ldots, \ell-1$ we have:

$$
\begin{aligned}
\left(m_{k}, \ldots, m_{k+2 \ell-1}\right) & =\left(m_{k}^{*}, \ldots, m_{k+2 \ell-1}^{*}\right), \\
\operatorname{clock}(\operatorname{sid}, k+2 i) & <\operatorname{clock}\left(\operatorname{sid}^{*}, k+2 i\right), \\
\text { and } \quad \operatorname{clock}(\operatorname{sid}, k+2 i+1) & >\operatorname{clock}\left(\operatorname{sid}^{*}, k+2 i+1\right) .
\end{aligned}
$$

As shown in Figure 2, our notion is slightly conservative. We account for computation complexity depending on input values, allowing adversaries to receive one reply, change the response, and relay it in time. But now adversaries could flip redundant bits and relay crucial ones without tainting a phase. We nonetheless prefer to err on the safe side and give adversary more freedom, as obvious redundancy is easily modified as shown for key exchange protocols $[7,6]$. Secondly, time-critical phases are tainted if all transmitted messages are relayed in two sessions. However, if a single transmission is relayed, the phase is untainted. Here we give adversaries more freedom and get a stronger notion.

The adversary must now make the reader accept in session sid such that for each adversary-tag session sid* at most $T_{\max }$ phases of sid are tainted by sid*:

Definition 3.2 (Mafia Fraud Resistance) For a distance-bounding identification scheme $\mathcal{I D}$ with parameters $\left(t_{\max }, T_{\max }, E_{\max }, N_{c}\right)$, a $\left(t, q_{\mathcal{R}}, q_{\mathcal{T}}, q_{\mathrm{OBS}}\right)$-Mafia-fraud adversary $\mathcal{A}$ wins against $\mathcal{I D}$ if the reader accepts in a reader-adversary session sid such that any

are robust with respect to this idea, but this contradicts the implementation of reliable time measurements and enable denial-of-service attacks. 


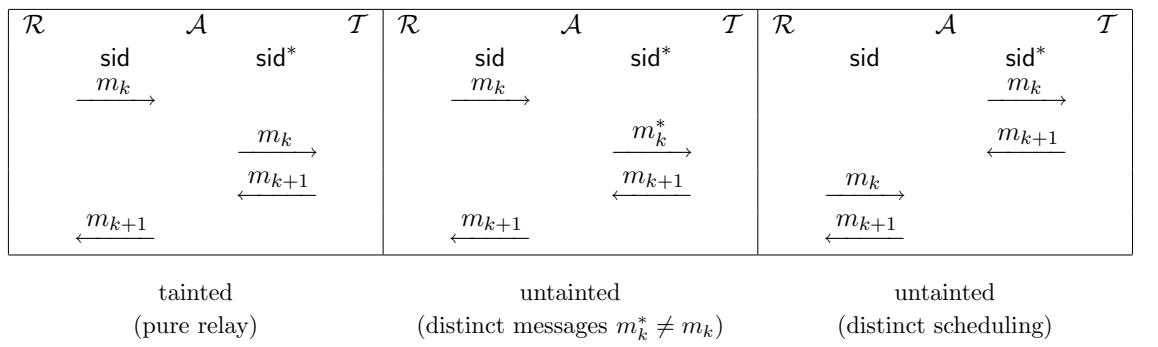

Figure 2: Examples of Tainted and Untainted Time-Critical Phases.

adversary-tag session sid $^{*}$ taints at most $T_{\text {max }}$ time-critical phases of sid. Let $\boldsymbol{A d v}_{\mathcal{I D}}^{\text {mafia }}(\mathcal{A})$ denote the probability that $\mathcal{A}$ wins.

Different adversary-tag sessions may taint different rounds of reader-adversary session sid. As we count $T_{\max }$ over all adversary-tag sessions the adversary wins if it taints at most $T_{\max }$ distinct phases. Protocols must prevent such attacks to be Mafia fraud secure in concurrent settings. Further session interdependencies should also be avoided so that messages from another session do not taint sid.

\subsection{Terrorist Attack Model}

In a terrorist attack the tag aids the adversary in all short of revealing its secret key, in fact wanting to ensure that the adversary only wins with the tag's aid (the dishonest prover controls the adversary's access). Desmedt [16] concretely describes the tag's involvement as offline help in a single impersonation attempt. The adversary now wins if the reader accepts, but the adversary cannot use the help given by tag $\mathcal{T}^{\prime}$ to impersonate further.

We formalize the idea by using ideas from proofs of computational ability $[38,5]$, which exactly capture the intuition of terrorist attacks: given support from a prover e.g. $\mathcal{T}^{\prime}$, one can solve a hard problem e.g. identifying to the reader. This is independent of how the prover gives support. We are not, however, interested in the cases where $\mathcal{T}^{\prime}$ yields the entire key (or large parts of it) and mark certain auxiliary data given by $\mathcal{T}^{\prime}$ as trivial, i.e. the data is trivial if it allows one to successfully complete a "fresh" identification attempt without help from $\mathcal{T}^{\prime}$. This includes the case when $\mathcal{T}^{\prime}$ gives the secret key, but circumvents the problem of determining which parts of the key are helpful. Data is trivial if it aids identification beyond the dedicated help in the session where $\mathcal{T}^{\prime}$ helps.

We formalize the latter by demanding that no algorithm $\mathcal{S}$, called simulator, can use the data passed by $\mathcal{T}^{\prime}$ to $\mathcal{A}$ to authenticate without the help of $\mathcal{T}^{\prime}$ (to be fair, we allow $\mathcal{S}$ the same number $q_{\mathcal{R}}$ of attempts as $\mathcal{A}$ ). This is in line with well-known simulation paradigms, and allows to compare the respective success probabilities of the adversary $\mathcal{A}$ aided by $\mathcal{T}^{\prime}$, and the simulator $\mathcal{S}$ using $\mathcal{A}$ 's information to authenticate. If $\mathcal{A}$ is significantly more successful than $\mathcal{S}$, the attack is non-trivial and the protocol is insecure against terrorist attacks. Note that "unsophisticated" adversaries may do worse than simulators for secure schemes, thus yielding negative advantages. 
For terrorist fraud, $\mathcal{A}$ acts as for Mafia fraud, but may query the "malicious" interface $\mathcal{T}^{\prime}$ in lazy phases. Sessions sid' with $\mathcal{T}^{\prime}$ are arbitrary, not following protocol.In fact we may consider only one session sid' when $\mathcal{T}^{\prime}$ helps $\mathcal{A}$. The tag may not aid $\mathcal{A}$ in time-critical phases, a fact which we model by defining tainted time-critical phases as pure-relay phases or rounds where $\mathcal{A}$ queries $\mathcal{T}^{\prime}$.

Definition 3.3 (Tainted Time-Critical Phase (Terror)) A time-critical phase $\Pi_{\text {sid }}[k \ldots k+$ $2 \ell-1]=\left(m_{k}, \ldots, m_{k+2 \ell-1}\right)$ for $k, \ell \geq 1$ of a reader-adversary session sid, with the $k$-th message being received by the adversary, is tainted if there is a session sid' between the adversary and $\mathcal{T}^{\prime}$ such that, for some $i$,

$$
\operatorname{clock}(\operatorname{sid}, k)<\operatorname{clock}\left(\operatorname{sid}^{\prime}, i\right)<\operatorname{clock}(\operatorname{sid}, k+2 \ell-1) .
$$

For the new definition of tainted phases, terrorist fraud resistance demands that for any terrorist fraud attacker $\mathcal{A}$ there exists a simulator $\mathcal{S}$ such that for any supporting $\mathcal{T}^{\prime}, \mathcal{S}$ is essentially as successful as $\mathcal{A}$. We use concrete security statements and omit quantification over $\mathcal{A}, \mathcal{S}$, and $\mathcal{T}^{\prime}$ algorithms; this quantification is included in subsequent security claims in the usual form (i.e., for any adversary there exists a simulator such that for all tags the advantage is small).

Definition 3.4 (Terrorist Fraud Resistance) Let $\mathcal{I D}$ be a distance-bounding identification scheme with parameters $\left(t_{\max }, T_{\max }, E_{\max }, N_{c}\right)$. Let $\mathcal{A}$ be a $\left(t, q_{\mathcal{R}}, q_{\mathcal{T}}^{\prime}\right)$-terroristfraud adversary, $\mathcal{S}$ be an algorithm running in time $t_{\mathcal{S}}$, and $\mathcal{T}^{\prime}$ be an algorithm running in time $t^{\prime}$. Denote

$$
\boldsymbol{A d v}_{\mathcal{I D}}^{\text {terror }}\left(\mathcal{A}, \mathcal{S}, \mathcal{T}^{\prime}\right)=p_{\mathcal{A}}-p_{\mathcal{S}}
$$

where $p_{\mathcal{A}}$ is the probability that the reader accepts in one of the $q_{\mathcal{R}}$ reader-adversary sessions sid such that at most $T_{\max }$ time-critical phases of sid are tainted, and $p_{\mathcal{S}}$ is the probability that, given view $w_{\mathcal{A}}$ in an attack of $\mathcal{A}, \mathcal{S}$ makes the reader accept in one of $q_{\mathcal{R}}$ subsequent executions.

Again, if the advantage is negative, $\mathcal{A}$ performs worse than $\mathcal{S}$. Our notion is quite strong: the simulator only gets to see $\mathcal{A}$ 's transcript in an offline phase, instead of communicating with $\mathcal{T}^{\prime}$ online. This guarantees stronger security and saves us from dealing with issues related to the number of queries and successful attacks (adversary vs. simulator).

How does our definition fit into previous efforts? Previous protocols [34, 4] claim to achieve a security of $(1 / 2)^{-N_{c}}$. This, however, corresponds to a tailor-made strategy of $\mathcal{T}^{\prime}$; other strategies may still exist. Proving that the advantage in Definition 3.4 is negligible, then we prove that $\mathcal{T}^{\prime}$ can only help trivially.

\subsection{Distance-Fraud Model}

For distance fraud an adversary must reply ahead of a time-critical phase or it cannot respond in time. In practice this is enforced by a tight value of $t_{\max }$. For any time-critical phase, with possibly many communication rounds, the adversary must commit to the first 
message to be sent. For any later rounds in the phase, the adversary has time to reply even from farther away.

The order of committed and sent values is determined by on oracle CommitTo with a single session sid CommitTo, taking tuples $\left(\mathrm{sid}, i, m_{i}\right)$ from the adversary and giving empty responses. The adversary commits to the first message of time-critical phase $i$ of session sid (message $j$ in sid) at time clock $\left(\operatorname{sid}_{\text {CommitTo }}, j\right.$ ). As the adversary may repeatedly commit to this message, we take the last commitment before phase $i$ begins.A time-critical phase is tainted if the adversary returns an answer it has not committed to.

Definition 3.5 (Tainted Time-Critical Phase (Distance)) A time-critical phase $\Pi_{\text {sid }}[k \ldots k+$ $2 \ell-1]=\left(m_{k}, \ldots, m_{k+2 \ell-1}\right)$ for $k, \ell \geq 1$ of a reader-adversary session sid, with the $k$-th message being received by the adversary, is tainted if the maximal $j$ with $\Pi_{\text {sid }}$ Commitтo $[j]=$ $\left(\right.$ sid, $\left.k+1, m_{k+1}^{*}\right)$ for some $m_{k+1}^{*}$ and clock(sid, $\left.k\right)>\operatorname{clock}(\operatorname{sid}$ CommitTo, $j)$ satisfies $m_{k+1}^{*} \neq$ $m_{k+1}$ (or no such $j$ exists).

Definition 3.6 (Distance Fraud Resistance) For an identification scheme $\mathcal{I D}$ with parameters $\left(t_{\max }, T_{\max }, E_{\max }, N_{c}\right), a\left(t, q_{\mathcal{R}}, q_{\mathcal{T}}, q_{\mathrm{OBS}}\right)$-distance-fraud adversary $\mathcal{A}$ wins against $\mathcal{I D}$ if the reader accepts in one of $q_{\mathcal{R}}$ reader-adversary sessions sid with at most $T_{\max }$ tainted time-critical phases. Let $\boldsymbol{A} \boldsymbol{d} \boldsymbol{v}_{\mathcal{I D}}^{\text {dist }}(\mathcal{A})$ be the probability of $\mathcal{A}$ winning.

\subsection{Impersonation Resistance}

We suggest a simple, but very strong definition of impersonation security as a basic requirement of identification in our concurrent setting. Thus even adversaries who actively take part in intertwined prover and verifier runs cannot impersonate the prover. Whereas the previous properties concern time-critical phases, impersonation security requires that an adversary cannot impersonate a tag in lazy phases. This ensures that the reader leaks no time-critical information to an invalid tag. Following the idea that parties should authenticate even if the time-critical phases are not executed, we consider projections $\Pi_{\text {sid }}^{\text {lazy }}[1 \ldots]$ of $\Pi_{\text {sid }}[1 \ldots]$ containing lazy phases transmissions only, and (not necessarily consecutive) indices $\iota_{\text {sid }}^{\text {lazy }}=\left(i_{1}, i_{2}, \ldots\right)$ of lazy phase messages. The adversary wins if a reader-adversary session succeeds and no adversary-tag session has the same "lazy transcript", created via pure relaying.

Definition 3.7 (Impersonation Resistance) In a distance-bounding identification scheme $\mathcal{I D}$ with parameters $\left(t_{\max }, T_{\max }, E_{\max }, N_{c}\right)$ where $\mathcal{R}$ always go first, a $\left(t, q_{\mathcal{R}}, q_{\mathcal{T}}, q_{\mathrm{OBS}}\right)$ impersonation adversary $\mathcal{A}$ wins against $\mathcal{I D}$ if $\mathcal{R}$ accepts in a reader-adversary session sid such that no adversary-tag session sid* has

$$
\Pi_{\text {sid }}^{l a z y}[1 \ldots]=\Pi_{\text {sid }^{*}}^{l a z y}[1 \ldots],
$$

and

$$
\operatorname{clock}(\operatorname{sid}, i)<\operatorname{clock}\left(\operatorname{sid}^{*}, i\right)
$$


for any $i \in \iota_{\text {sid }}^{\text {lazy }} \cap \iota_{\text {sid* }}^{\text {lazy }}$ s.t. $\mathcal{R}$ has sent the $i$-th message to $\mathcal{A}$ in sid, and

$$
\operatorname{clock}(\operatorname{sid}, j)>\operatorname{clock}\left(\operatorname{sid}^{*}, j\right)
$$

for any $j \in \iota_{\text {sid }}^{\text {lazy }} \cap \iota_{\text {sid* }}^{\text {lazy }}$ such that the adversary has sent the $j$-th message to the reader in sid. Let $\boldsymbol{A} \boldsymbol{d} \boldsymbol{v}_{\mathcal{I D}}^{i m p}(\mathcal{A})$ be the probability that $\mathcal{A}$ wins.

\section{Relationship between Fraud Types}

Impersonation security concerns lazy protocol phases, while Terrorist, Mafia, and distance fraud attack time-critical phases. In our framework we refute the idea in [34] that terrorist fraud resistance implies distance fraud resistance and show that all properties are independent. Due to limited space, we leave the formal proofs for the full version and give only an intuition below.

Theorem 4.1 (Security Diagram - Informal) If pseudorandom functions exist, the following holds:

1. There exists a distance-bounding identification scheme that is impersonation-secure, Mafia and distance fraud resistant, but not terrorist fraud resistant.

2. There exists a distance-bounding identification scheme that is impersonation-secure, Terrorist and Mafia fraud resistant, but not distance fraud resistant. Thus, terrorist fraud resistance does not imply distance fraud resistance.

3. There exists a distance-bounding identification scheme that is impersonation-secure, Terrorist and distance fraud resistant, but not Mafia fraud resistant. Thus, terrorist fraud resistance does not imply Mafia fraud resistance.

Terrorist-Fraud Resistance. The enhanced Kim-Avoine scheme in Section 5 has all properties except for terrorist-fraud resistance. The reason it fails against terrorist attacks is that time-critical messages are predetermined by the lazy phase and can be revealed without disclosing the secret key (thus providing sufficient, but non-trivial offline help). In general, terrorist attacks are thwarted by interlinking authentication sessions, such that malicious tags (partially) reveal long-term secrets if they help the adversary. The difficulty in designing terrorist-fraud resistant schemes is formally ensuring that the simulator can extract the secret from the adversary and thus authenticate. The simulator's only advantage is that it can rewind executions and get responses for different challenges.

Distance-Fraud Resistance. We separate distance-fraud resistance from the other properties by giving the tag a special key which makes time-critical responses predictable. Honest parties never use this key, but malicious tags may use it to commit distance fraud. Other security properties are unaffected, as the special key is never used by honest parties. Distance-fraud resistance depends on the unpredictability of each round's answer. This is easily achieved by adding some time-critical rounds where tags echo random bits. 
Mafia-Fraud Resistance. We show Mafia fraud resistance independence by starting with a protocol having all other security properties; the tag may use a bit to indicate that time-critical bits are flipped. Then a man-in-the-middle adversary can flip replies from an adversary-tag session and authenticate to the reader without tainting the phases. There are two options to prevent Mafia fraud attacks. Assume that in each fast phase the reader sends a random challenge. If the adversary correctly predicts the challenge in a reader impersonation, it can use the reply in the reader-adversary session without tainting the phase; for a wrong prediction, the adversary guesses the answer instead. The overall success is $\frac{3}{4}$ per round as in, e.g., the Hancke and Kuhn protocol [26]. The other option is to authenticate the reader by the fast phase challenges. Now the adversarytag session in the above attack aborts for a wrong prediction, dropping the adversary's success probability in the reader-adversary execution to $\frac{1}{2}$ for subsequent rounds. This is the strategy of the Kim-Avoine as discussed next.

\section{Case Study: The Construction due to Kim and Avoine}

The scheme in [29] is Mafia and Distance fraud resistant. We tweak it to add impersonation security, provide for noisy channels as in Section 2, then prove it secure in our framework. The proof relies on the fact that the nonce pairs exchanged in each run are quasi unique; also for any efficient adversary $\mathcal{A}^{\prime}$ the advantage $\operatorname{Adv}_{\mathrm{PRF}}^{\operatorname{dist}}\left(\mathcal{A}^{\prime}\right)$ of distinguishing a pseudorandom function from a truly random one is small (see Appendix $\mathrm{A}$ for a formal proof).

Theorem 5.1 (Security Properties) The distance-bounding identification scheme ID in Fig. 3 with parameters $\left(T_{\max }, t_{\max }, E_{\max }, N_{c}\right)$ has the following properties:

- It is not terrorist-fraud resistant.

- For any $\left(t, q_{\mathcal{R}}, q_{\mathcal{T}}, q_{\mathrm{OBS}}\right)$-impersonation adversary $\mathcal{A}$ against $\mathcal{I} \mathcal{D}$ there exists a $\left(t^{\prime}, q^{\prime}\right)$ distinguisher $\mathcal{A}^{\prime}$ against PRF (with $t^{\prime}=t+O(n)$ and $q^{\prime}=q_{\mathcal{R}}+q_{\mathcal{T}}+q_{\mathrm{OBS}}$ ) such that,

$$
\boldsymbol{A} \boldsymbol{d} \boldsymbol{v}_{\mathcal{I D}}^{i m p}(\mathcal{A}) \leq q_{\mathcal{R}} \cdot 2^{-|I|}+\boldsymbol{A} \boldsymbol{d} \boldsymbol{v}_{\mathrm{PRF}}^{\text {dist }}\left(\mathcal{A}^{\prime}\right)+\left(\begin{array}{c}
q_{\mathcal{R}}+q_{\mathrm{OBS}} \\
2
\end{array}\right) \cdot 2^{-\left|N_{\mathcal{R}}\right|}+\left(\begin{array}{c}
q_{\mathcal{T}} \\
2
\end{array}\right) \cdot 2^{-\left|N_{\mathcal{T}}\right|}
$$

- For any $\left(t, q_{\mathcal{R}}, q_{\mathcal{T}}, q_{\mathrm{OBS}}\right)$-distance-fraud adversary $\mathcal{A}$ against $\mathcal{I D}$ there is a $\left(t^{\prime}, q^{\prime}\right)$ distinguisher $\mathcal{A}^{\prime}$ against PRF (where $t^{\prime}=t+O(n)$ and $q^{\prime}=q_{\mathcal{R}}+q_{\mathcal{T}}+q_{\mathrm{OBS}}$ ) such that, for $N_{t}=T_{\max }+E_{\max }$

$$
\boldsymbol{A} \boldsymbol{d} \boldsymbol{v}_{\mathcal{I} \mathcal{D}}^{\text {dist }}(\mathcal{A}) \leq q_{\mathcal{R}} \cdot\left(\begin{array}{l}
N_{c} \\
N_{t}
\end{array}\right)\left(\frac{7}{8}\right)^{N_{c}-N_{t}}+\boldsymbol{A} \boldsymbol{d} \boldsymbol{v}_{\mathrm{PRF}}^{\text {dist }}\left(\mathcal{A}^{\prime}\right)+\left(\begin{array}{c}
q_{\mathcal{R}}+q_{\mathrm{OBS}} \\
2
\end{array}\right) \cdot 2^{-\left|N_{\mathcal{R}}\right|}
$$

- For any $\left(t, q_{\mathcal{R}}, q_{\mathcal{T}}, q_{\mathrm{oBS}}\right)$-Mafia-fraud adversary $\mathcal{A}$ against $\mathcal{I D}$ there exists a $\left(t^{\prime}, q^{\prime}\right)$ distinguisher $\mathcal{A}^{\prime}$ against PRF (where $t^{\prime}=t+O(n)$ and $q^{\prime}=q_{\mathcal{R}}+q_{\mathcal{T}}+q_{\mathrm{OBS}}$ ) such 
that, for $N_{t}=T_{\max }+2 E_{\max }$

$$
\begin{array}{r}
\boldsymbol{A d v}_{\mathcal{I D}}^{\text {mafia }}(\mathcal{A}) \leq \frac{5}{8} \cdot q_{\mathcal{R}}\left(\begin{array}{c}
N_{c} \\
N_{t}
\end{array}\right) \cdot\left(N_{c}-N_{t}+2\right) \cdot 2^{-\left(N_{c}-N_{t}\right)}+\boldsymbol{A} \boldsymbol{d} \boldsymbol{v}_{\mathrm{PRF}}^{\text {dist }}\left(\mathcal{A}^{\prime}\right) \\
+\left(\begin{array}{c}
q_{\mathcal{R}}+q_{\mathrm{OBS}} \\
2
\end{array}\right) \cdot 2^{-\left|N_{\mathcal{R}}\right|}+\left(\begin{array}{c}
q_{\mathcal{T}} \\
2
\end{array}\right) \cdot 2^{-\left|N_{\mathcal{T}}\right|}
\end{array}
$$



Figure 3: Enhanced Kim/Avoine protocol.

For a single impersonation attempt and $T_{\max }=E_{\max }=0$ we have up to small terms the (almost optimal) bound $\frac{1}{2}\left(N_{c}+2\right) \cdot 2^{-N_{c}}$ for Mafia-Fraud resistance. The distance fraud resistance of $\frac{7}{8}$ per round is tight, corresponding to an adversary who sends $v_{i}^{0}$ in round $i$ ( $v^{0}$ is precomputed in the lazy phase).

\section{References}

[1] Abyneh, M.R.S.: Security analysis of two distance-bounding protocols. In: Proceedings of RFIDSec 2011. Lecture Notes in Computer Science, Springer (2011) 
[2] Avoine, G., Bingol, M.A., Karda, S., Lauradoux, C., Martin, B.: A formal framework for analyzing rfid distance bounding protocols. In: Journal of Computer Security Special Issue on RFID System Security, 2010 (2010)

[3] Avoine, G., Martin, B., Martin, T.: Optimal security limits of rfid distance bounding protocols. In: RFIDSec 2010. pp. $220-238$

[4] Avoine, G., Tchamkerten, A.: An efficient distance bounding rfid authentication protocol: Balancing false-acceptance rate and memory requirement. In: Information Security. Lecture Notes in Computer Science, vol. 5735, pp. 250-261. Springer-Verlag (2009)

[5] Bellare, M., Goldreich, O.: Proving computational ability. http://www.wisdom.weizmann.ac.il/ oded/PS/poa.ps (1992)

[6] Bellare, M., Pointcheval, D., Rogaway, P.: Authenticated key exchange secure against dictionary attacks. In: Advances in Cryptology - Eurocrypt 2000. Lecture Notes in Computer Science, vol. 1807, pp. 139-155. Springer-Verlag (2000)

[7] Bellare, M., Rogaway, P.: Entity authentication and key distribution. In: Advances in Cryptology - Crypto '93. Lecture Notes in Computer Science, vol. 773, pp. 232249. Springer-Verlag (1994)

[8] Brands, S., Chaum, D.: Distance-bounding protocols. In: Advances in Cryptology - Eurocrypt'93. pp. 344-359. Lecture Notes in Computer Science, Springer-Verlag (1993)

[9] Bringer, J., Chabanne, H.: Trusted-hb: A low-cost version of hb ${ }^{+}$secure against man-in-the-middle attacks. Transactions on Information Theory 54(9), 4339-4342 (2008)

[10] Bussard, L., Bagga, W.: Distance-bounding proof of knowledge to avoid real-time attacks. Security and Privacy in the Age of Ubiquitous Computing 181, 222-238 (2005)

[11] Camenisch, J., Lysyanskaya, A.: An efficient system for non-transferable anonymous credentials with optional anonymity revocation. In: Advances in Cryptology - Eurocrypt. Lecture Notes in Computer Science, vol. 2045, pp. 93-118. Springer-Verlag (2001)

[12] Capkun, S., Butty'an, L., Hubaux, J.P.: Sector: Secure tracking of node encounters in multi-hop wireless networks. In: ACM Workshop on Security of Ad Hoc and Sensor Networks - SASN. pp. 21 - 32. ACM Press (2003)

[13] Carluccio, D., Kasper, T., Paar, C.: Implementation details of a multi purpose iso 14443 rfidtool. In: Printed handout of Workshop on RFID Security - RFIDSec 06 (July 2006) 
[14] Chandran, N., Goyal, V., Moriarty, R., Ostrovsky, R.: Position based cryptography. In: Advances in Cryptology - Crypto. Lecture Notes in Computer Science, vol. 5677, pp. 391-407. Springer-Verlag (2009)

[15] Clulow, J., Hancke, G.P., Kuhn, M.G., Moore, T.: So near and yet so far: Distancebounding attacks in wireless networks. In: European Workshop on Security and Privacy in Ad-Hoc and Sensor Networks. Lecture Notes in Computer Science, vol. 4357, pp. 83-97. Springer-Verlag (2006)

[16] Desmedt, Y.: Major security problems with the 'unforgeable' (feige)-fiat-shamir proofs of identity and how to overcome them. In: SecuriCom. pp. 15-17. SEDEP Paris, France (1988)

[17] Drimer, S., Murdoch, S.J.: Keep your enemies close: distance bounding against smartcard relay attacks. In: Proc. of the 16-th USENIX Security Symposium on USENIX Security Symposium, article no. 7. ACM Press (2007)

[18] Duc, D., Kim, K.: Securing hb+ against grs man-in-the-middle attack. In: Symposium on Cryptography and Information Security (SCIS). The Institute of Electronics, Information and Communication Engineers (2007)

[19] Francillon, A., Danev, B., Capkun, S.: Relay attacks on passive keyless entry and start systems in modern cars. Cryptology ePrint Archive, Report 2010/332 (2010), ePRINTURL

[20] Gilbert, H., Robshaw, M., Sibert, H.: An active attack against hb+ - a provably secure lightweight authentication protocol. Cryptology ePrint Archive, Report 2005/237 (2005), ePRINTURL

[21] Goldreich, O., Pfitzmann, B., Rivest, R.L.: Self-delegation with controlled propagation - or - what if you lose your laptop. In: Advances in Cryptology - Crypto. Lecture Notes in Computer Science, vol. 1462, pp. 153-168. Springer-Verlag (1998)

[22] Haataja, K., Toivanen, P.: Two practical man-in-the-middle attacks on bluetooth secure simple pairing and countermeasures. Transactions on Wireless Communications 9(1), 384-392 (2010)

[23] Hancke, G.P.: A practical relay attack on iso 14443 proximity cards. http://www.cl.cam.ac.uk/gh275/relay.pdf (2005)

[24] Hancke, G.: Distance bounding publication database. http://www.rfidblog.org.uk/db.html (2010)

[25] Hancke, G.P.: Design of a secure distance-bounding channel for rfid. Journal of Network and Computer Applications (2010)

[26] Hancke, G.P., Kuhn, M.G.: An rfid distance bounding protocol. In: SECURECOMM. pp. 67-73. ACM Press (2005) 
[27] Hopper, N.J., Blum, M.: Secure human identification protocols. In: Proceedings of the 7th International Conference on the Theory and Application of Cryptology and Information Security ADVCRYPTO. Lecture Notes in Computer Science, vol. 2248, pp. 52-66. Springer-Verlag (2001)

[28] Juels, A.: Rfid security and privacy: a research survey. IEEE Journal on Selected Areas in Communications 24(2), 381-394 (2006)

[29] Kim, C.H., Avoine, G.: Rfid distance bounding protocol with mixed challenges to prevent relay attacks. In: Proceedings of the 8th International Conference on Cryptology and Networks Security (CANS 2009). Lecture Notes in Computer Science, vol. 5888, pp. 119-131. Springer-Verlag (2009)

[30] Leng, X., Mayes, K., Markantonakis, K.: Hb-mp+ protocol: An improvement on the hb-mp protocol. In: International Conference on RFID. pp. 118-124. IEEE Computer Society Press (2008)

[31] Meadows, C., Poovendran, R., Pavlovic, D., Chang, L., Syverson, P.: Distance bounding protocols: Authentication logic analysis and collusion attacks. In: Proceedings of Secure Localization and Time Synchronization for Wireless Sensor and Ad Hoc Networks. Springer-Verlag (2007)

[32] Ouafi, K., Overbeck, R., Vaudenay, S.: On the security of hb\# against a man-inthe-middle attack. In: Advances in Cryptology - Asiacrypt 2008. Lecture Notes in Computer Science, vol. 5350, pp. 108-124. Springer (2008)

[33] Rasmussen, K.B., Čapkun, S.: Realization of rf distance bounding. USENIX Security Symposium (2010)

[34] Reid, J., Nieto, J.M.G., Tang, T., Senadji, B.: Detecting relay attacks with timingbased protocols. In: ASIACCS. pp. 204-213. ACM Press (2007)

[35] Schaller, P., Schmidt, B., Basin, D., Capkun, S.: Modeling and verifying physical properties of security protocols for wireless networks. In: Proceedings of the 22nd IEEE Computer Security Foundations Symposium 2009. pp. 109-123. ACM (2009)

[36] Singele, D., Preneel, B.: Distance bounding in noisy environments. In: European Workshop on Security in Ad-hoc and Sensor Networks - ESAS. Lecture Notes in Computer Science, vol. 4572, pp. 101 - 115. IEEE Computer Society Press (2007)

[37] Trujillo-Rasua, R., Martin, B., Avoine, G.: The poulidor distance-bounding protocol. In: RFIDSec 2010. pp. $239-257$

[38] Yung, M.: Zero-knowledge proofs of computational power. In: Advances in Cryptology - Eurocrypt '89. Lecture Notes in Computer Science, vol. 434, pp. 196-207. Springer-Verlag (1990) 


\section{A Security Proof of the Protocol of Kim and Avoine}

Proof. The protocol is not terrorist-fraud resistant: $\mathcal{T}^{\prime}$ can forward adversary $\mathcal{A}$ the value $I\|C\| S\left\|v^{0}\right\| v^{1}$. Now $\mathcal{A}$ authenticates successfully; a simulator can't authenticate, however, as a fresh session has new nonces in the lazy phase.

We prove Mafia-fraud resistance as follows: (1) honest parties' PRF output by independent random values $I\|C\| D\left\|v^{0}\right\| v^{1}$ for new nonces $\left(N_{\mathcal{R}}, N_{\mathcal{T}}\right) ;(2)$ show quasiuniqueness of nonce pairs except in 1 adversary-tag session and 1 reader-adversary session s.t. $\mathcal{A}$ relays the nonces; (3) bound $\mathcal{A}$ 's winning probability in time-critical phases for at most one adversary-tag interaction.

Due to space limits, we only sketch steps (1) and (2). In (1), replacing PRF-values by random (but consistent) values decreases $\mathcal{A}$ 's success probability by at most the distinguishing advantage for PRF (else we use $\mathcal{A}$ to distinguish PRF). For (2), if $\mathcal{A}$ does not relay nonces, the probability of colliding nonces is

$$
\left(\begin{array}{c}
q_{\mathcal{R}}+q_{\mathrm{oBS}} \\
2
\end{array}\right) \cdot 2^{-\left|N_{\mathcal{R}}\right|}+\left(\begin{array}{c}
q_{\mathcal{T}} \\
2
\end{array}\right) \cdot 2^{-\left|N_{\mathcal{T}}\right|}
$$

Now let $\mathcal{A}$ lose if the nonces match apart from the case above. Now the values $I\|C\| \mid D\left\|v^{0}\right\| v^{1}$ are independent. Let sid be a reader-adversary session where $\mathcal{A}$ successfully impersonates to $\mathcal{R}$. By assumption at most one other adversary-tag session sid* has the same nonce pair. If sid* exists, it taints sid with high probability (if sid* doesn't exist, $\mathcal{A}$ can't benefit from sid*). Suppose now that sid* taints at most $T_{\text {max }}$ time-critical phases of sid. Assume for the moment that $E_{\max }=0$; we make provisions for $E_{\max }>0$ later.

Consider an untainted time-critical phase of sid where $\mathcal{R}$ sends $R_{i}$ and expects $T_{i}$, i.e. assume $\mathcal{A}$ successfully passed the first $i-1$ time-critical phases. There are four strategies for the adversary in this $i$-th phase:

Go-EARLy. In session sid* $\mathcal{A}$ sends bit $R_{i}^{*}$ to $\mathcal{T}$ before receiving $R_{i}$ (i.e., clock(sid, $\left.i+2\right)>$ clock $\left.\left(\operatorname{sid}^{*}, i+2\right)\right)$. As $R_{i}$ is random and independently chosen, $R_{i}^{*} \neq R_{i}$ w.p. $\frac{1}{2}-$ then $\mathcal{A}$ doesn't receive $T_{i}$ in sid* and must guess $T_{i}$ in sid. Also, session sid* becomes invalid with probability $\frac{1}{4}$.

Go-LATE. In session sid, $\mathcal{A}$ replies to $R_{i}$ with $T_{i}$ before receiving $T_{i}^{*}$ in session sid* $\left(\operatorname{clock}(\operatorname{sid}, i+3)<\operatorname{clock}\left(\operatorname{sid}^{*}, i+3\right)\right)$. Now $\mathcal{A}$ wins the phase w.p. $\frac{1}{2}$.

Modify-IT. $\mathcal{A}$ receives $R_{i}$ in sid, sends $R_{i}^{*}$ in sid*, gets $T_{i}^{*}$ in sid*, and forwards $T_{i}$ in sid. This scheduling is pure relay, but $R_{i} \neq R_{i}^{*}$ or $T_{i} \neq T_{i}^{*}$. If $R_{i}^{*}$ is wrong then $T_{i}^{*}$ was never sent by $\mathcal{T}$ in sid* and $\mathcal{A}$ can only guess $T_{i}$ w.p. $\frac{1}{2}$; if $R_{i}=R_{i}^{*}$ then $T_{i} \neq T_{i}^{*}$ makes the reader reject.

TAINT-IT. The adversary taints this phase of sid through sid*.

Tainting the phase makes $\mathcal{R}$ accept with probability 1 , deducting 1 from the remaining taintable phases. The Go-Late and Modify-it Strategy both succeed w.p. at most $\frac{1}{2}$. 
Go-Early succeeds w.p. $\frac{3}{4}$, inactivating sid* w.p. $\frac{1}{2}$. Assume that $\mathcal{A}$ taints the last $T_{\max }$ time-critical phases (else we renumber the phases). For the other $P:=N_{c}-T_{\max }$ phases let pass $_{i}$ denote the event that $\mathcal{A}$ passes phase $i$ of sid. We have

$\operatorname{Prob}\left[\bigwedge_{j=i}^{P} \operatorname{pass}_{j} \mid \bigwedge_{j=1}^{i-1} \operatorname{pass}_{j}\right] \leq \frac{5}{8} \cdot \operatorname{Prob}\left[\bigwedge_{j=i+1}^{P} \operatorname{pass}_{j} \mid \bigwedge_{j=1}^{i} \operatorname{pass}_{j}\right]+\frac{1}{2} \cdot \frac{1}{2} \cdot 2^{-P+i+1}$

The first term captures the success of Go-Late, Modify-It, and correct Go-Early-prediction. The second term covers incorrect Go-Early prediction (w.p. $\frac{1}{4}$ ); now sid* is inactivated, and $\mathcal{A}$ must guess $T_{i}$ for this and the next $P-i-1$ rounds (the responses are independent). Expanding the probabilities we obtain

$$
\operatorname{Prob}\left[\bigwedge_{j=1}^{P} \text { pass }_{j}\right] \leq 2^{-P}+\sum_{j=0}^{P-1} \frac{5}{8} \cdot 2^{-j} \cdot 2^{-P+j}=\frac{5}{8} \cdot(P+2) \cdot 2^{-P} .
$$

We sum over $q_{\mathcal{R}}$ reader-adversary sessions, distribute $T_{\max }+E_{\max }$ "jokers" on the reader side and $E_{\max }$ on the tag side, and obtain the claimed bound.

For impersonation security, the only way to generate colliding nonce pairs (and produce authentication string $I$ ) is by lazy phase relay, which is an invalid impersonation attack. For distinct nonce pairs, the probability that $\mathcal{A}$ sends a correct $I$ in a readeradversary session is: $q_{\mathcal{R}} \cdot 2^{-|I|}$ plus the distinguishing advantage for the PRF plus the probability of colliding nonces.

Distance-bounding (the third statement) is proved as above: once the pseudorandom values are replaced by truly random ones, the probability that $C_{i}=1$ and $v_{i}^{0} \neq v_{i}^{1}$ is at least $\frac{1}{4}$ for round $i$. Since $\mathcal{A}$ can commit only then, $\mathcal{A}$ fails with probability at least $\frac{1}{8}$. Overall, $\mathcal{A}$ succeeds only w.p. $\frac{7}{8}$ per round, except for a number $T_{\max }+E_{\max }$ of phases. 